\title{
Shellability and the strong gcd-condition
}

\author{
Alexander Berglund* \\ Department of Mathematics \\ Stockholm University, Sweden \\ alexb@math.su.se
}

Submitted: Aug 13, 2008; Accepted: Feb 3, 2009; Published: Feb 11, 2009

Mathematics Subject Classification: 55U10, 13F55

\begin{abstract}
Shellability is a well-known combinatorial criterion on a simplicial complex $\Delta$ for verifying that the associated Stanley-Reisner ring $k[\Delta]$ is Cohen-Macaulay. A notion familiar to commutative algebraists, but which has not received as much attention from combinatorialists as the Cohen-Macaulay property, is the notion of a Golod ring. Recently, Jöllenbeck introduced a criterion on simplicial complexes reminiscent of shellability, called the strong gcd-condition, and he together with the author proved that it implies Golodness of the associated Stanley-Reisner ring. The two algebraic notions were earlier tied together by Herzog, Reiner and Welker, who showed that if $k\left[\Delta^{\vee}\right]$ is sequentially Cohen-Macaulay, where $\Delta^{\vee}$ is the Alexander dual of $\Delta$, then $k[\Delta]$ is Golod. In this paper, we present a combinatorial companion of this result, namely that if $\Delta^{\vee}$ is (non-pure) shellable then $\Delta$ satisfies the strong gcd-condition. Moreover, we show that all implications just mentioned are strict in general but that they are equivalences if $\Delta$ is a flag complex.
\end{abstract}

\section{To Anders Björner on his sixtieth birthday}

\section{Introduction}

Let $\Delta$ be a finite simplicial complex with vertex set $V=\left\{v_{1}, \ldots, v_{n}\right\}$ and let $k$ be a field. Recall that the Stanley-Reisner ring associated to $\Delta$ is the quotient

$$
k[\Delta]=k\left[x_{1}, \ldots, x_{n}\right] / I_{\Delta},
$$

where $I_{\Delta}$ is the ideal in the polynomial ring $k\left[x_{1}, \ldots, x_{n}\right]$ generated by the monomials $x_{i_{1}} \ldots x_{i_{r}}$ for which $\left\{v_{i_{1}}, \ldots, v_{i_{r}}\right\} \notin \Delta$. The Cohen-Macaulay property of Stanley-Reisner

\footnotetext{
${ }^{*}$ Current affiliation: Department of Mathematical Sciences, University of Copenhagen, Denmark. Email: alexb@math.ku.dk
} 
rings has been intensely studied, and this has led to several important results in combinatorics. See the book [12] for an overview. The generalized concept of sequentially Cohen-Macaulay rings will play a role here, for the definition see [12, Definition III.2.9].

A ring of the form $R=k\left[x_{1}, \ldots, x_{n}\right] / I$, where $I \subseteq\left(x_{1}, \ldots, x_{n}\right)^{2}$, is called a Golod ring if all Massey operations on the Koszul complex $K\left(x_{1}, \ldots, x_{n}, R\right)$ ([12, Definition 2.39]) vanish, see [7, Definition 4.2.5]. There are several equivalent but differently flavored characterizations of Golod rings, see Sections 5.2 and 10.3 in [1] and the references therein. One is that $R$ is Golod if the ranks of the modules in a minimal free resolution of the $R$-module $k \cong R /\left(x_{1}, \ldots, x_{n}\right)$ have the fastest possible growth, see [1, p.42]. A reason for being interested in knowing that a ring $R$ is Golod is that then one can write down explicitly a minimal free resolution of $k$, see [1, Theorem 5.2.2]. Golodness of StanleyReisner rings can be characterized in terms of poset homology, see [2, Theorem 3]. See also [3], [4] for some recent work on the Golod property of Stanley-Reisner rings.

We will say that a simplicial complex $\Delta$ is sequentially Cohen-Macaulay, or Golod, if the Stanley-Reisner ring $k[\Delta]$ has that property. Not much more will be said about these algebraic notions, but we will be interested in their combinatorial companions: shellability and the strong gcd-condition. Let us begin by recalling their definitions. If $F_{1}, \ldots, F_{r} \subseteq V$ then let

$$
\left\langle F_{1}, \ldots, F_{r}\right\rangle
$$

denote the simplicial complex generated by $F_{1}, \ldots, F_{r}$. It consists of all subsets $F \subseteq V$ such that $F \subseteq F_{i}$ for some $i$.

Definition 1 (Björner, Wachs [5]). A (not necessarily pure) simplicial complex $\Delta$ is called shellable if the facets of $\Delta$ admit a shelling order. A shelling order is a linear order, $F_{1}, \ldots, F_{r}$, of the facets of $\Delta$ such that for $2 \leq i \leq r$, the simplicial complex

$$
\left\langle F_{i}\right\rangle \cap\left\langle F_{1}, \ldots, F_{i-1}\right\rangle
$$

is pure of dimension $\operatorname{dim}\left(F_{i}\right)-1$.

As is well-known and widely exploited, shellability is a combinatorial criterion for verifying that a pure complex is Cohen-Macaulay. The notion of sequentially CohenMacaulay complexes, due to Stanley, was conceived as a non-pure generalization of the notion of Cohen-Macaulay complexes that would make the following proposition true:

Proposition 2 (Stanley [12]). Every shellable simplicial complex is sequentially CohenMacaulay.

We now move to the strong ged-condition.

Definition 3 (Jöllenbeck [10]). A simplicial complex $\Delta$ is said to satisfy the strong gcd-condition if the set of minimal non-faces of $\Delta$ admits a strong gcd-order. A strong gcd-order is a linear order, $M_{1}, \ldots, M_{r}$, of the minimal non-faces of $\Delta$ such that whenever $1 \leq i<j \leq r$ and $M_{i} \cap M_{j}=\emptyset$, there is a $k$ with $i<k \neq j$ such that $M_{k} \subseteq M_{i} \cup M_{j}$. 
The strong gcd-condition was introduced because of its relation to the Golod property. In [10], Jöllenbeck made a conjecture a consequence of which was that the strong gcdcondition is sufficient for verifying that a complex is Golod. One of the main results of the paper [4] was a proof of that conjecture, thus establishing the truth of the next proposition.

Proposition 4 (Berglund, Jöllenbeck [4]). A simplicial complex satisfying the strong gcd-condition is Golod.

The following result ties together the notions of sequentially Cohen-Macaulay rings and Golod rings, via the Alexander dual. Recall that the Alexander dual of $\Delta$ is the simplicial complex

$$
\Delta^{\vee}=\left\{F \subseteq V \mid F^{c} \notin \Delta\right\} .
$$

Here and henceforth $F^{c}$ denotes the complement of $F$ in $V$. The facets of $\Delta^{\vee}$ are the complements in $V$ of the minimal non-faces of $\Delta$.

Proposition 5 (Herzog, Reiner, Welker [9]). If the Alexander dual $\Delta^{\vee}$ is sequentially Cohen-Macaulay then $\Delta$ is Golod.

What we have said so far can be summarized by the following diagram of implications:



This diagram seems to indicate that the strong gcd-condition plays the same role for the Golod property as shellability does for the property of being sequentially CohenMacaulay. What we wish to do next is to tie together the accompanying combinatorial notions by proving the implication represented by the dashed arrow. After that, we will give examples of simplicial complexes, $\Delta_{1}, \Delta_{2}$ and $\Delta_{3}$, having the following configurations of truth values in the diagram:

\begin{tabular}{|cc|cc|cc|}
\hline \multicolumn{2}{|c|}{$\Delta_{1}$} & \multicolumn{2}{|c|}{$\Delta_{2}$} & \multicolumn{2}{|c|}{$\Delta_{3}$} \\
\hline$F$ & $T$ & $F$ & $T$ & $F$ & $F$ \\
$F$ & $T$ & $T$ & $T$ & $F$ & $T$ \\
\hline
\end{tabular}

In particular, all implications in the diagram are strict. However, we will finish by proving that if $\Delta$ is a flag complex, then all arrows are in fact equivalences.

\section{Weak shellability}

We think of the set of vertices $V$ as part of the data in specifying a simplicial complex, so potentially there could be 'ghost vertices', i.e., vertices $v \in V$ such that $\{v\} \notin \Delta$. Requiring that $\Delta$ has no ghost vertices is equivalent to requiring that $|F| \leq|V|-2$ for all facets $F$ of $\Delta^{\vee}$. The Stanley-Reisner ring $k[\Delta]$ does not see ghost vertices in the sense that $k[\Delta] \cong k\left[\Delta^{\prime}\right]$, where $\Delta^{\prime}$ is the complex $\Delta$ with ghost vertices removed. 
Proposition 6. Let $\Delta$ be a simplicial complex without ghost vertices. If $\Delta^{\vee}$ is shellable then $\Delta$ satisfies the strong gcd-condition.

Proof. Let $F_{1}, \ldots, F_{r}$ be a shelling order of the facets of $\Delta^{\vee}$. The minimal non-faces of $\Delta$ are then $F_{1}^{c}, \ldots, F_{r}^{c}$. We claim that the reversed order, $F_{r}{ }^{c}, \ldots, F_{1}{ }^{c}$, is a strong gcdorder for $\Delta$. By the assumption that $\Delta$ has no ghost vertices, $\left|F_{i}{ }^{c}\right| \geq 2$, or in other words $\left|F_{i}\right| \leq|V|-2$, for all $i$.

Let $1 \leq i<j \leq r$ and suppose that $F_{i}^{c} \cap F_{j}^{c}=\emptyset$. We must produce a $k$ with $i \neq k<j$ such that $F_{k}{ }^{c} \subseteq F_{i}^{c} \cup F_{j}^{c}$. The assumption means that $F_{i} \cup F_{j}=V$. Combining this with the fact $\left|F_{i}\right| \leq|V|-2$, we get

$$
\left|F_{i} \cap F_{j}\right| \leq\left|F_{j}\right|-2 .
$$

Since $F_{1}, \ldots, F_{r}$ is a shelling order, the complex

$$
\left\langle F_{j}\right\rangle \cap\left\langle F_{1}, \ldots, F_{j-1}\right\rangle
$$

is pure of dimension $\operatorname{dim}\left(F_{j}\right)-1$. Of course, $F_{i} \cap F_{j}$ is contained in this complex. Let $H$ be a facet of the complex containing $F_{i} \cap F_{j}$. Then $|H|=\left|F_{j}\right|-1$. If $H \subseteq F_{i}$, then $H \subseteq F_{i} \cap F_{j}$, but this is impossible since $\left|F_{i} \cap F_{j}\right| \leq\left|F_{j}\right|-2$. Therefore, $H$ is contained in some $F_{k}$ where $i \neq k<j$. Hence, $F_{i} \cap F_{j} \subseteq H \subseteq F_{k}$, which implies that $F_{k}^{c} \subseteq F_{i}^{c} \cup F_{j}^{c}$. This finishes the proof.

By using the correspondence between minimal non-faces of $\Delta$ and facets of $\Delta^{\vee}$, one can rephrase the strong gcd-condition as a property of $\Delta^{\vee}$ in the following way:

Definition 7. A simplicial complex $\Delta$ is called weakly shellable if the facets of $\Delta$ admit a weak shelling order. A weak shelling order is a linear order, $F_{1}, \ldots, F_{r}$ of the facets of $\Delta$ such that if $1 \leq i<j \leq r$ and $F_{i} \cup F_{j}=V$ then there is a $k$ with $i \neq k<j$ such that $F_{i} \cap F_{j} \subseteq F_{k}$.

Then the following is clear by definition:

Proposition 8. Let $\Delta$ be a simplicial complex and let $M_{1}, \ldots, M_{r}$ be its minimal nonfaces. Then the facets of $\Delta^{\vee}$ are $F_{i}=M_{i}^{c}, i=1, \ldots, r$, and the order $M_{1}, \ldots, M_{r}$ is a strong gcd-order if and only if $F_{r}, F_{r-1}, \ldots, F_{1}$ is a weak shelling order.

In fact, the proof of Proposition 6 shows the following:

Proposition 9. Let $\Delta$ be a simplicial complex such that $|F| \leq|V|-2$ for all $F \in \Delta$. Then any shelling order of the facets of $\Delta$ is a weak shelling order.

Remark 10. Note that if $\Delta$ is a $d$-dimensional simplicial complex with $|V| \geq 2 d+3$, then $\Delta$ is automatically weakly shellable because in this case $|F \cup G|<|V|$ for all faces $F, G \in$ $\Delta$. In particular, by subdividing an arbitrary simplicial complex $\Delta$ enough times one obtains a weakly shellable complex whose geometric realization is homeomorphic to the one of $\Delta$. Thus, any triangulable space can be triangulated by a weakly shellable simplicial complex. This is in contrast to the well-known fact that the geometric realization of a shellable simplicial complex is homotopy equivalent to a wedge of spheres. However, one might ask whether or not weakly shellable complexes with $|V|<2 d+3$ have some special topological property. 


\section{$3 \quad$ Examples}

Example 11. Let $\Delta_{1}$ be the simplicial complex with vertex set $\{1,2,3,4,5,6\}$ and minimal non-faces $\{1,2,3\},\{1,2,6\},\{4,5,6\}$. The Alexander dual $\Delta_{1}^{\vee}$ has facets $\{1,2,3\}$, $\{3,4,5\},\{4,5,6\}$, and it is not Cohen-Macaulay because the link of the vertex 3 is onedimensional but not connected. However, the order in which the minimal non-faces of $\Delta_{1}$ appear above is in fact a strong gcd-order.

Example 12. Let $\Delta_{2}^{\vee}$ be the triangulation of the 'dunce hat' with vertices $1,2, \ldots, 8$ and facets

$$
\begin{array}{r}
\{1,2,4\},\{1,2,7\},\{1,2,8\},\{1,3,4\},\{1,3,5\},\{1,3,6\},\{1,5,6\},\{1,7,8\},\{2,3,5\} \\
\{2,3,7\},\{2,3,8\},\{2,4,5\},\{3,4,8\},\{3,6,7\},\{4,5,6\},\{4,6,8\},\{6,7,8\} .
\end{array}
$$

It is well-known that any triangulation of the dunce hat is Cohen-Macaulay but not shellable. Furthermore, for this particular triangulation, $|V|=8 \geq 7=2 \operatorname{dim}\left(\Delta_{2}^{\vee}\right)+3$, so $\Delta_{2}^{\vee}$ is automatically weakly shellable, which means that $\Delta_{2}$ satisfies the strong gcdcondition.

Example 13. Let $\Delta_{3}$ be the simplicial complex with vertices $0,1, \ldots, 9$ and minimal non-faces

$$
\{0,1,5,6\},\{1,2,6,7\},\{2,3,7,8\},\{3,4,8,9\},\{0,4,5,9\},\{5,6,7,8,9\} .
$$

One can check by a direct computation that this simplicial complex is Golod. However, the strong gcd-condition is violated because for each 3-dimensional minimal non-face $M$ there are two 3-dimensional minimal non-faces $M^{\prime}$ and $M^{\prime \prime}$ with $M^{\prime} \cap M^{\prime \prime}=\emptyset$ and such that $M$ is the unique minimal non-face different from $M^{\prime}$ and $M^{\prime \prime}$ with $M \subseteq M^{\prime} \cup M^{\prime \prime}$. In other words, there is no way of deciding which of these $M$ should come first in a strong gcd-order.

Next, if the dual complex $\Delta_{3}^{\vee}$ were sequentially Cohen-Macaulay, then by [12, Proposition III.2.10] the pure subcomplex $\Gamma$ generated by the facets of maximum dimension would be Cohen-Macaulay. However, $\Gamma$ has facets

$$
\{0,1,2,5,6,7\},\{0,1,4,5,6,9\},\{0,3,4,5,8,9\},\{2,3,4,7,8,9\},
$$

and the link $L=\operatorname{lk}_{\Gamma}\{0,1,5,6\}$ has facets $\{2,7\}$ and $\{4,9\}$, so $L$ is one-dimensional but disconnected, and therefore $\Gamma$ is not Cohen-Macaulay.

The reader might wonder why we have not provided an example with the table

$$
\begin{array}{ll}
F & F \\
T & T
\end{array}
$$

The Alexander dual of a simplicial complex having this table would need to be a nonshellable sequentially Cohen-Macaulay complex with $|V|<2 d+3$. Already finding complexes meeting these specifications seems difficult: All but one of the examples of nonshellable Cohen-Macaulay complexes found in [8] satisfy $|V| \geq 2 d+3$, and are therefore 
weakly shellable for trivial reasons. The exception is the classical 6-vertex triangulation of the real projective plane, which is however easily seen to be weakly shellable. Also, Gräbe's example [6] of a complex which is Gorenstein when the characteristic of the field $k$ is different from 2 but not Gorenstein otherwise is weakly shellable. It has been shown that all 3-balls with fewer than 9 vertices are extendably shellable, and that all 3 -spheres with fewer than 10 vertices are shellable, see [11], so there is no hope in finding an example there. The author would however be very surprised if no example existed.

Problem 14. Find a sequentially Cohen-Macaulay complex which is not weakly shellable.

\section{$4 \quad$ Flag complexes}

Recall that a flag complex is a simplicial complex all of whose minimal non-faces have two elements. Order complexes associated to partially ordered sets are important examples of flag complexes. Note that the Alexander dual of a flag complex is pure, and for pure complexes sequentially Cohen-Macaulay means simply Cohen-Macaulay.

Proposition 15. Suppose that $\Delta$ is a flag complex. Then the following are equivalent:

(1) $\Delta^{\vee}$ is shellable.

(2) $\Delta$ satisfies the strong gcd-condition.

(3) $\Delta^{\vee}$ is Cohen-Macaulay.

(4) $\Delta$ is Golod.

Proof. For the equivalence of (2), (3) and (4), see [4, Theorem 4]. The implication (1) $\Rightarrow$ (2) follows from Proposition 6. What remains to be verified is the implication $(2) \Rightarrow(1)$ and this is contained in the next proposition.

Proposition 16. If $\Delta$ is a flag complex then any weak shelling order of the facets of $\Delta^{\vee}$ is a shelling order.

Proof. Let $F_{1}, \ldots, F_{r}$ be a weak shelling order of the facets of $\Delta^{\vee}$. The complements $F_{1}{ }^{c}, \ldots, F_{r}{ }^{c}$ are the minimal non-faces of the flag complex $\Delta$, so $\left|F_{i}{ }^{c}\right|=2$ and $\left|F_{i}\right|=|V|-2$ for all $i$. Let $j \geq 2$ and consider the complex

$$
\left\langle F_{j}\right\rangle \cap\left\langle F_{1}, \ldots, F_{j-1}\right\rangle \text {. }
$$

We want to show that it is pure of dimension $\operatorname{dim}\left(F_{j}\right)-1=|V|-4$. The facets therein are the maximal elements in the set of all intersections $F_{i} \cap F_{j}$, where $i<j$. Clearly, $\left|F_{i} \cap F_{j}\right| \leq|V|-3$, since otherwise $F_{i}=F_{i} \cap F_{j}=F_{j}$. Suppose that $\left|F_{i} \cap F_{j}\right| \leq|V|-4$. We will show that $F_{i} \cap F_{j}$ is not maximal. Indeed, we have that

$$
|V|-4 \geq\left|F_{i} \cap F_{j}\right|=\left|F_{i}\right|+\left|F_{j}\right|-\left|F_{i} \cup F_{j}\right|=2|V|-4-\left|F_{i} \cup F_{j}\right|,
$$


which implies that $\left|F_{i} \cup F_{j}\right| \geq|V|$, whence $F_{i} \cup F_{j}=V$. By the definition of a weak shelling order, there is a $k$ with $i \neq k<j$ such that $F_{i} \cap F_{j} \subseteq F_{k}$. Say $F_{i}^{c}=\left\{v_{i}, w_{i}\right\}$, $F_{j}{ }^{c}=\left\{v_{j}, w_{j}\right\}$ and $F_{k}{ }^{c}=\left\{v_{k}, w_{k}\right\}$. Then $\left\{v_{k}, w_{k}\right\} \subseteq\left\{v_{i}, w_{i}, v_{j}, w_{j}\right\}$. Since the facets $F_{i}$ and $F_{k}$ are distinct either $v_{k}$ or $w_{k}$ is in $\left\{v_{j}, w_{j}\right\}$. This means that $\left|F_{k}{ }^{c} \cup F_{j}{ }^{c}\right| \leq 3$, that is, $\left|F_{k} \cap F_{j}\right| \geq|V|-3$. Hence $F_{i} \cap F_{j}$ is a proper subset of $F_{k} \cap F_{j}$, so it is not maximal.

Acknowledgements. The author would like to thank two anonymous referees for helpful suggestions.

\section{References}

[1] L. L. Avramov, Infinite free resolutions, Six lectures on commutative algebra (Bellaterra, 1996), 1-118, Progr. Math., 166, Birkhäuser, Basel, 1998.

[2] A. Berglund, Poincaré series of monomial rings, J. Algebra 295 (2006), no.1, 211230.

[3] A. Berglund, J. Blasiak, P. Hersh, Combinatorics of multigraded Poincaré series for monomial rings, J. Algebra 308 (2007), no.1, 73-90.

[4] A. Berglund, M. Jöllenbeck, On the Golod property of Stanley-Reisner rings, J. Algebra 315 (2007), no. 1, 249-273.

[5] A. Björner, M. Wachs, Shellable nonpure complexes and posets. I, Trans. Amer. Math. Soc. 348 , No. 4, (1996), 1299-1327.

[6] H.-G. Gräbe, The Gorenstein property depends on characteristic, Beiträge Algebra Geom. 17 (1984), 169-174.

[7] T. H. Gulliksen, G. Levin, Homology of local rings, Queen's Paper in Pure and Applied Mathematics, No. 20 Queen's University, Kingston, Ont. 1969.

[8] M. Hachimori, Simplicial complex library, http://infoshako.sk.tsukuba.ac.jp/\%7Ehachi/math/library/index_eng.html

[9] J. Herzog, V. Reiner, V. Welker, Componentwise linear ideals and Golod rings, Mich. Math. J. 46 (1999), 211-223.

[10] M. Jöllenbeck, On the multigraded Hilbert- and Poincaré series of Monomial Rings, J. Pure Appl. Algebra 207, No.2, (2006), p. 261-298.

[11] F. H. Lutz, Combinatorial 3-manifolds with 10 vertices, Beiträge Algebra Geom. 49 (2008), no. 1, 97-106.

[12] R. Stanley, Combinatorics and Commutative Algebra, 2nd ed., Progress in Math., vol. 41, 1996, Birkhäuser Boston Inc. 